\title{
Transient High-frequency Impedance Comparison Based Protection for Flexible DC Distribution Systems
}

\author{
Ke Jia, Member, IEEE, Zhenwen Xuan, Tao Feng, Congbo Wang, Tianshu Bi, Senior Member, IEEE \\ and David Thomas, Senior Member, IEEE
}

\begin{abstract}
Flexible direct current (DC) distribution systems have emerged as the development trend for future distribution grids. However, these systems are vulnerable to DC faults, rapid fault identification and faulted line selection method are required to enhance the security of the entire system. A novel transient highfrequency impedance comparison based DC protection for flexible DC distribution systems is proposed in this paper. The controlindependent high frequency impedance model of power converter is also investigated. Based on this model, the proposed method identified the faulted lines by comparing high frequency impedance measurement differences. For DC bus with multiple branches, this technique minimizes the threshold calculation job, which is usually difficult to process for the transient value based protections. Strict synchronization of data is also not required for this method. The simulation model of four-terminal flexible DC distribution networks is built in PSCAD/EMTDC to verify the effectiveness of the proposed protection model. Simulation results prove that the protection is robust to fault transition resistances and the measurement noise.
\end{abstract}

Index Terms - Flexible DC grid, modular multilevel converter, high-frequency impedance, directional pilot protection

\section{INTRODUCTION}

$\mathrm{W}$ ith widespread permeation of the distributed energies and DC loads, the traditional AC distribution network needs more power conversion stages and breakers with higher breaking capacity. The DC distribution system can reduce the power commutation link and not increase the fault current on the AC side. In addition, the DC network is more suitable for cable-transmission[1], which can alleviate the land shortage in modern city. The larger power supply radius and the excellent power quality make it become the popular development trend for future power distribution grids[2-6]. However, the system is vulnerable to DC fault. When a pole-to-pole fault occurs in the flexible DC distribution system, the fault current will rise rapidly [7], seriously damaging the power converter and other electronic devices in the system. Therefore, it is imperative to identify faults rapidly, and selectively isolate the fault point by DC protection. In previously published papers, the protection applied in DC distribution systems could be divided into the following two categories: 1) Time domain measurement based DC protections; 2) Frequency domain measurement based DC protections.

The DC protections[8-9] which directly utilize the time domain data can be further divided into two categories depending on whether the communications are required or not. These are non-unit protections and unit protections. Non-unit protections can identify the DC faults using locally measured

This work was supported by the State's Key Project of Research and Development Plan under the Grant 2018YFB0904100, the NSFC project (51725702, 51777071), and Young Elite Scientists Sponsorship Program by CAST 2018QNRC001. information[10], such as overcurrent protection discussed in Ref.[11], current derivative based DC protections (di/dt, $\left.d^{2} i / d t^{2}\right)$, and voltage derivative based protections $(d u / d t)$ which were investigated respectively in [12-14]. However, applying a suitable threshold setting for the above protections may require a high level of the operation experience. Authors of the literatures [15-17] constructed a clear protected boundary by installing reactors at both terminals of the DC line. It is relatively easy to partition the DC line faults using these techniques by comparing the transient characteristics when the fault is located in different positions (between the two reactors or external). However, the additional DC reactors will reduce the power transformation efficiency and deteriorate the performance of control system that might not be available for all of the DC distributions [18]. Traveling-wave (TW) based protection introduced in reference [19-20] can rapidly discriminate the DC faults. However, the TW-based protections will suffer malfunctions or refused to operate when the fault is located very close to the measuring devices [21]. Furthermore, the TW-based protection is mainly equipped in the transmission systems especially the point-to- point transmission line. For the DC distribution network with multiple branches, the TW-based protection can be challenged due to wide reflection of the travelling wave. Unit protections with the communication naturally have clear boundaries, for example, current differential protection can locate DC faults rapidly and selectively [22]. However, measuring information at the both terminals of the DC line is required, and the data synchronization for both sides must be strictly carried out [2324]. In reference [25], directional and over-current criteria is adopted to determine the directions of the fault and carry out fault identification via applying the directional pilot protection. This method does not require the strict data synchronization, however, its operation performance can be challenged by the oscillation of DC fault currents.

For frequency domain based protections, the authors in [26] calculated the current amplitude at the resonant frequencies of the DC filter to identify the faults. The impedance angle was calculated under the resonant frequencies of the DC filters to carry out internal/external fault identification in [27]. However, the protection schemes proposed in [26-27] are mainly applied in the conventional DC transmission systems, which mostly utilize an inductive DC filter to smooth voltage waveforms. For the flexible DC system, the DC filter is unnecessary due to its excellent power quality. Thus, such protections would not be suitable to the flexible DC system. In [28], accurate fault area/pole identification is carried out by measuring transient impedance. This method demonstrates excellent performance when the filter-boundary exists, but it would be compromised when used in the multi-branch system investigated in this study. 
Reference [29-30] analyzed the AC-side high-frequency impedance characteristics of the Two-level VSC converter and further propose a high-frequency impedance measuring based fault location method. However, for DC distribution system, the high-frequency impedance model is inapplicable and needs to be rebuilt according to specific converter topology (MMC, DC/DC converter) and the fault types (DC fault). Paper [31] proposed a wavelet-transform (WT) based DC fault detection method via calculating Pearson correlation coefficient between the wavelet and fault pattern, the faults can be detected using local measurements. Ref [32] investigated a STFT-based DC fault detection strategy. The fault is identified according to the distortion of frequency spectrums. Comparing with WT-based method, the STFT-methods are more efficient in computation speed. However, for multi-terminal system, the faulted line is required to be further selected out to trigger the specific relays. The fault detection and location method based on the WT are investigated in the Ref [33-34]. In paper [35], the faulted line could be discriminated by comparing the wavelet coefficients with the setting thresholds which are determined by multiple simulations. The discrete wavelet transform (DWT) magnitude is calculated in Ref [34] to discriminate the faulted line with the inductive terminals. For the investigated DC system, there may be no line reactors to form the inductive boundary.

A novel unit protection utilizing the high frequency domain information to identify the DC pole to pole faults rapidly and selectively is proposed in this paper. When a DC fault occurs, a deep voltage sag will occur at the fault point, which can be seen as the superposition of a large number of high-frequency signals with attenuation characteristics. Power converters can be linearized into a constant impedance in the high frequency band, regardless of different control strategies. Faults located at different positions can be distinguished using comparisons of the measured transient high-frequency impedances. As the high-frequency signal source is located at the fault point, the proposed protection remains robust when experiencing fault transition resistance compared with traditional protections. On the other hand, the high-frequency impedance model of power converter is investigated which simply performs as a constant impedance. Therefore, the proposed protection is only slightly affected by different converter control schemes. The proposed method also does not require complexed threshold setting in the case of multiple line connection, the strict synchronization of data measurement is unnecessary and is low-bandwidth for the data communication. In addition, the sensors are equipped around the DC bus rather than on the both ends of each branch comparing with traditional pilot protection, which is beneficial for the multi-branch system with distributed energy integration. To verify the effectiveness of proposed protection algorism, a four-terminal flexible DC distribution system has been built in PSCAD/EMTDC platform.

The remainder of this paper is structured as follows: in the Section II, an analysis of the transient high-frequency signals generation mechanism and the control-independent impedance model of the converter in the high frequency band is presented, which is the basis of proposed protection. Section III details the novel fault identification criterion. Section IV verifies the performance of the new protection under different influencing factors. Finally, conclusions are drawn in Section V.

\section{TRANSIENT HIGH-FREQUENCY IMPEDANCE ANALYSIS}

\section{A. Structure of the Investigated DC Distribution System}

The investigated flexible DC distribution system, as shown in Fig. 1, is composed of the modular multilevel converters (MMC) and DC/DC converters. The MMC converters adopt a special topology based on clamp double sub-module (CDSM), which can clear DC fault currents within $3 \mathrm{~ms}$ after the fault inception (the detailed topology of CDSM-MMC is present in subsection $\mathrm{C}$ ). The rated voltage of the DC distribution system is $\pm 10 \mathrm{kV}$ with the insulated neutral, and the AC system is grounded through the Z-type transformer. The symbol $f_{1} \sim f_{5}$ denotes the fault positions, 'SW' represents the fast isolators. (For the economic and applicable reasons, the fast isolators are used to remove the faulted line instead of the DC circuit breakers) The measuring device groups of '1 4' deployed on the DC bus (as shown in the dashed box) includes No. 1 10 units respectively connected with each of the DC lines to obtain line's voltages and currents. In the investigated system, the DCside neutral points of the two DC/DC converters are connected to the ground through a high-impedance $\left(10^{4} \Omega\right)$, the transient current is too weak to be detected and not damage the system when the DC pole-to-ground fault occurs. Therefore, this paper will mainly focus on the DC pole-to-pole faults which is required to be removed very fast.

\section{B. High-frequency Signal Generation Mechanism}

When DC fault occurs, the voltage of the fault point will suffer a deep sag that is similar to a step signal superimposed on the original DC voltage waveform, the step change and its spectrum can be expressed as (1)(2). Fig.2(a-d) demonstrate the transient DC voltage/current waveform and their spectrum extracted via the Continuous-Wavelet Transform (CWT) when

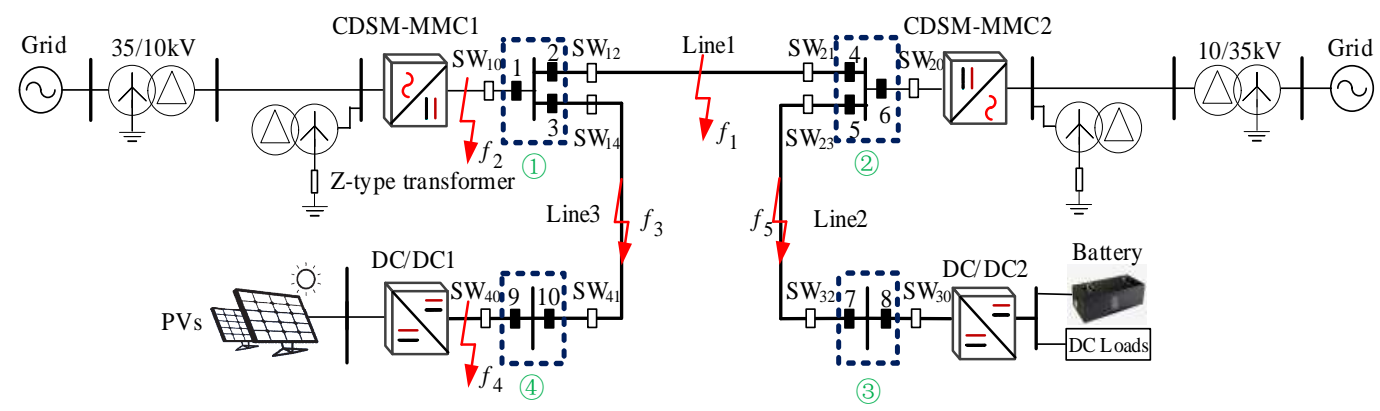

Fig. 1. Structure of the investigated DC distribution network 


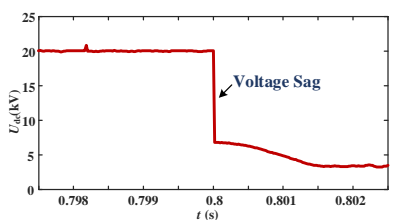

(a) The DC fault voltage waveform

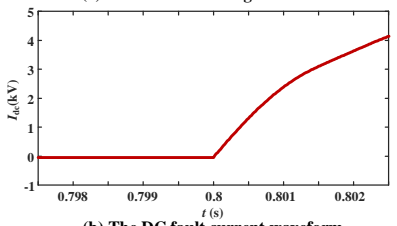

(b) The DC fault current waveform

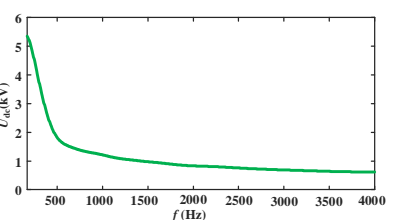

(c) The spectrum of $\mathrm{DC}$ fault voltage

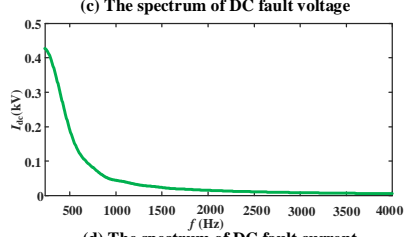

(d) The spectrum of DC fault current
Fig. 2. The fault voltage/current waveforms and their spectrums

the fault is located at $f_{1}$. It can be concluded that spectral value $|U(j \omega)|$ of the DC voltage sag are distributed over the entire frequency domain, its spectral energy is inversely proportional to the frequencies. Therefore, the DC fault transient voltage at the fault point will contain a large number of the frequency components with high signal-to-noise ratios. These frequency components can be seen as a signal source located at the fault point in protected line, and can be used for fault identification. Moreover, these frequency domain voltage and current signals can be precisely extracted by CWT as detailed in [35]. The transient impedance can be described in Laplace domain, as shown in (3), in which $\omega$ is the angular frequency, symbol $j$ denotes the imaginary unit.

$$
\begin{gathered}
\varepsilon(t)=\left\{\begin{array}{l}
1, t>0 \\
0, t<0
\end{array}\right. \\
F(j \omega)=\pi \delta(t)+\frac{1}{j \omega} \\
Z(\mathrm{~s})=U(\mathrm{~s}) / I(\mathrm{~s}) \Rightarrow Z(j \omega)=U(j \omega) / I(j \omega)
\end{gathered}
$$

Considering the converters will be blocked within $3 \mathrm{~ms}$ of the fault inception [36-37], only the data before the converter blocking is valid for the proposed method. The low-frequency signals are difficult to obtain accurately in such a narrow data window, while high-frequency information is easy to capture in this scenario. Moreover, the converter internal capacitors will behave the low-impedance characteristics compared with the bridge-arm reactors in the high frequency band. Thus, the converter DC-side equivalent circuit can be linearized into a constant impedance by characterizing it in the high-frequency domain (detailed analysis is provided in subsection $\mathrm{C}$ below). Therefore, impedance information under the high-frequency band will be investigated in this paper.

\section{The DC-side High-frequency Impedance of Converter}

The faulty converter is extremely difficult to model in time domain due to its unpredictable switching behavior under the fault. However, this can be simplified by characterizing it in the high-frequency band, in which the effect of the converter switching could be eliminated considering the low-impedance characteristic of sub-capacitors in high-frequency domain.

\section{i) High-frequency model of DC/DC converter}

For DC/DC converters in the investigated system, a large capacitor is parallel on the high-voltage side, as illustrated in the Appendix. Thus, the DC/DC converter will conduct the low-

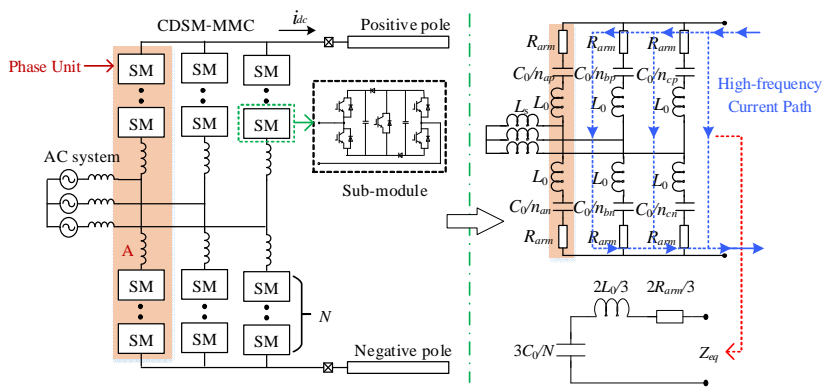

Fig. 3(a). DC-side high-frequency model of CDSM-MMC

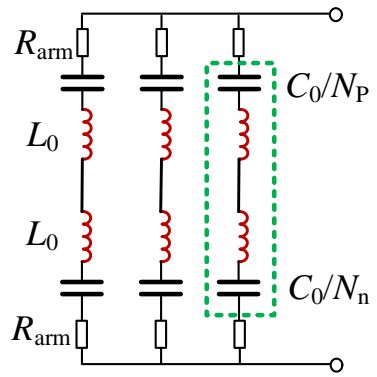

Fig. 3(b). The RLC circuit in a certain switching status

impedance characteristic to the high-frequency signal that is generated by the DC fault.

\section{ii) High-frequency model of CDSM-MMC}

For MMC-type converter, the sub-capacitors are distributed inside the bridge-arm, and there is a reactor connected with the sub-capacitor in series. This high-frequency impedance model will be dramatically different to DC/DC converters. A detailed topology of CDSM-MMC is presented in the Fig. 3(a), which includes three phase-units parallel connected between positive and negative poles. Each phase-unit is further composed of $2 \mathrm{~N}$ sub-modules and two bridge-arm reactors in series. To maintain a constant DC-bus voltage, the converter modulation will ensure the sum of 'on-state' sub-modules in each phase- unit holds a constant $N$ at any time. Thus, each bridge arm of MMC can be described as a $R L C$ circuit in a certain switching status in Fig.3(b). Where $L_{0}$ represents bridge-arm inductance, $C_{0}$ is capacitance of the sub-modules, $R_{\text {arm }}$ denotes bridge-arm resistance, and $n_{a p}, n_{a n}, n_{b p}, n_{b n}, n_{c p}, n_{c n}$ represent the number of the 'on-state' sub-module in each bridge arm.

Although the 'on-state' sub-modules number of each phase unit is dynamic and is determined by the switching status, the effects of converter's switching behavior can be eliminated in the high frequency band. It is mainly due to the sub-capacitor low-impedance characteristic in the high-frequency band.

According to the Fig. 3, since the high-frequency inductive impedance of the bridge-arm reactor is much larger than the sub-capacitors capacitive high-frequency impedance (4), each phase-units can be approximately replaced by the bridge-arm reactors, and the three phase-units are almost symmetrical to the high-frequency signal generated by the DC fault. Thus, the shunt effects of grid equivalent impedance $L_{\mathrm{s}}$ can be ignored, and the high-frequency model of the converter can be further simplified as a $R L C$ circuit. The total impedance of converter is derived in (5) as a constant contributed by the converter subcapacitors, bridge-arm reactors, and resistors. 


$$
\begin{gathered}
\omega L_{0}>N /\left(\omega C_{0}\right) \\
Z_{e q}=\frac{2}{3} R_{a r m}+j \frac{1}{3}\left(2 \omega L_{0}-N / \omega C_{0}\right)
\end{gathered}
$$

\section{Transient High-frequency Impedance Analysis}

The fault superimposed networks for the different DC fault positions are illustrated in Fig. 4. The signal source $U_{f}(j \omega)$ is located at fault point. Symbols $Z_{r 1} \sim Z_{r 4}$ denote the transient impedances in Laplace domain of DC/DC converters and the CDSM-MMC. Subscript $Z_{l i}$ represents the transient impedance of the No. $i$ DC line, and $Z_{l i j}$ denotes the transient impedance of $j$-th partition, which belongs to the No. $i$ DC line (Symbol ' $\perp$ ' in Fig. 4 represents the potential reference points rather than real grounding electrodes).

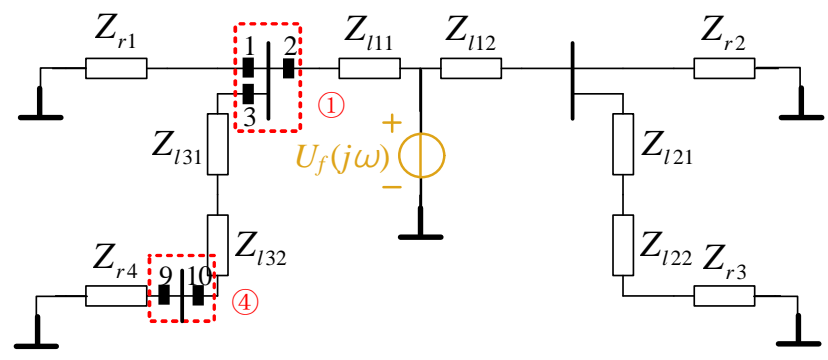

Fig. 4(a). High-frequency superimposed network of $f_{1}$ fault

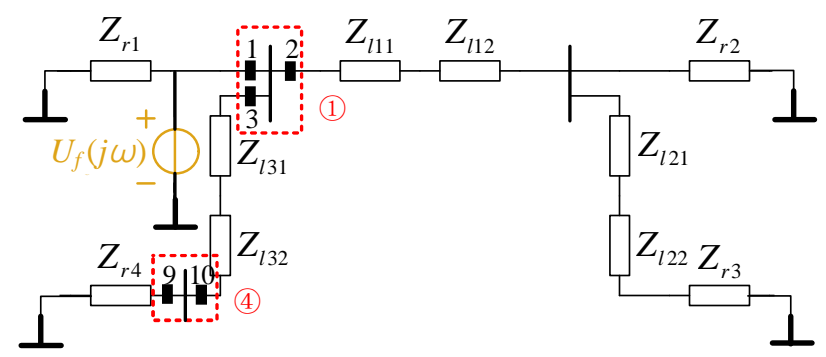

Fig. 4(b). High-frequency superimposed network of $f_{2}$ fault

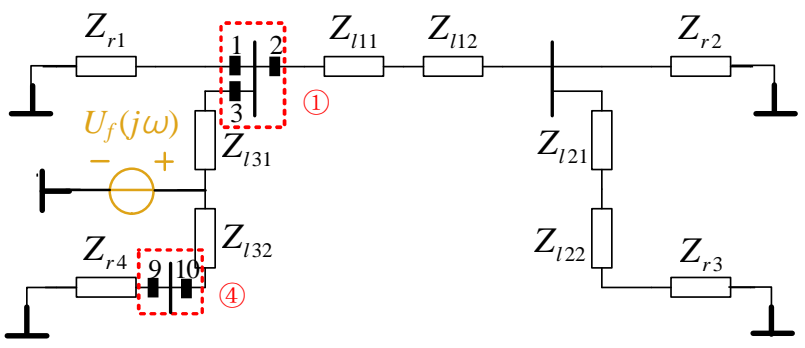

Fig. 4(c). High-frequency superimposed network of $f_{3}$ fault

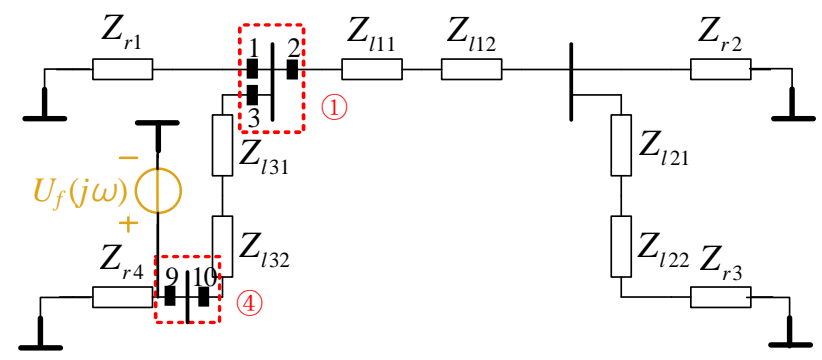

Fig. 4(d). High-frequency superimposed network of $f_{4}$ fault
Transient impedances in Fig. 4 are operational impedances in Laplace domain, and can be described by using magnitudefrequency curves. The analysis in the Section B proves that the signals in the high-frequency band are suitable to be used for protections. Thus, the transient high-frequency impedance is defined as the amplitude at a certain high-frequency point $\left(\omega_{k}\right)$ on the transient impedance magnitude-frequency curve.

When the fault is located at $f_{1}$. It can be seen from Fig.4(a), the theoretical high-frequency impedance of measuring unit 1 is $Z_{r 1}$ (high-frequency impedance of MMC-1). The theoretical measuring impedance of unit 3 is the series impedance of $Z_{r 4}$ and $Z_{l 3}$. (The sum of high-frequency impedance of DC/DC-1 $Z_{r 4}$ and high-frequency impedance of the DC Line $3 Z_{l 3}$ ). The theoretical measuring impedance of the unit 2 is the parallel one of the $Z_{1}$ and $Z_{2}$. These above theoretical high-frequency impedances of each measuring unit is described as (6):

$$
\left\{\begin{array}{l}
Z_{1}=Z_{r 1}\left(\omega_{k}\right) \\
Z_{2}=Z_{1}\left(\omega_{k}\right) / / Z_{3}\left(\omega_{k}\right) \\
Z_{3}=Z_{r 4}\left(\omega_{k}\right)+Z_{l 3}\left(\omega_{k}\right)
\end{array}\right.
$$

In (6), the theoretical high-frequency impedance of unit 2 is the parallel one of $Z_{1}$ and $Z_{3}\left(Z_{2}=Z_{1} / / Z_{3}\right)$. Considering the shunt impedance is less than that of any branch, the measuring highfrequency impedance of the unit 2 (directly connects with the faulted line) is the minimum in device group (1) as shown in (7):

$$
\left|Z_{2}\right|<\left|Z_{1}\right| \text { and }\left|Z_{2}\right|<\left|Z_{3}\right|
$$

When the fault is located at $f_{2}$. It can be seen in Fig.4(b), the theoretical measuring impedance of unit1 is the parallel one of $Z_{2}$ and $Z_{3}$. Therefore, the measuring transient high-frequency impedance of unit 1 will be the minimum in the device group (1) as shown in (8):

$$
\left|Z_{1}\right|<\left|Z_{2}\right| \text { and }\left|Z_{1}\right|<\left|Z_{3}\right|
$$

Similarly, when the DC fault is located at $f_{3}$ as shown in Fig. 4(c), the (9) can be obtained as following:

$$
\left|Z_{3}\right|<\left|Z_{1}\right| \text { and }\left|Z_{3}\right|<\left|Z_{2}\right|
$$

As seen from (7)-(9), the measuring units which holds the minimum transient high-frequency impedance are different in measuring device group (1) when the fault is located at the different positions. The measuring unit that directly connected with the faulted line will hold the minimum value of transient high-frequency impedance in the measuring device group due to the shunt effect. The fault direction can be determined by comparing these transient high-frequency impedances in the same measuring device group deployed on DC bus.

However, if the DC bus only has two branches, the shunt effect described in the (7)-(9) will not work. For instance, considering the measuring device group (4), the theoretical transient high-frequency impedance of the No. 9 and No. 10 measuring units can be obtained as formula (10-11), according to Fig. 4(c-d) when fault is located at $f_{3}$ and $f_{4}$, respectively:

$$
Z_{9}=Z_{10}=Z_{r 4}\left(\omega_{k}\right)
$$

$Z_{9}=Z_{10}=Z_{l 3}\left(\omega_{k}\right)+Z_{r 1}\left(\omega_{k}\right) / /\left[Z_{l 1}\left(\omega_{k}\right)+Z_{r 2}\left(\omega_{k}\right) / /\left(Z_{l 2}\left(\omega_{k}\right)+Z_{r 3}\left(\omega_{k}\right)\right)\right](11)$ 
Therefore, for the DC bus with only two lines connection, the measuring units deployed on each branch will hold equal transient high-frequency impedance for various fault locations. However, these values of transient high-frequency impedances vary for faults in different locations. The faulted line selection can be achieved by comparing the theoretical value and the measured value of the high-frequency impedances.

\section{PROPOSED DIRECTIONAL PILOT PROTECTION}

\section{A. Fault Identification Criterion}

When there are multiple (more than two) lines connected on the DC bus, as per the previous analysis, the high frequency impedance of fault-side measuring unit will be the minimum. In this scenario, the fault direction could be discriminated by comparing the high-frequency impedances in same measuring device group. In order to calculate high-frequency impedance $\mathrm{Z}_{i}^{*}\left(\omega_{k}\right)$, the amplitudes of voltage/current frequency-domain components $\left|U\left(j \omega_{k}\right)\right|$ and $\left|I\left(j \omega_{k}\right)\right|$ need to be extracted by the CWT from the time-domain sampling data, respectively. Then the high-frequency impedance at the frequency point $\omega_{k}$ can be obtained via the division of $\left|U\left(j \omega_{k}\right) /\right| I\left(j \omega_{k}\right) \mid$ in (3).

The root mean square (RMS) $A_{i}^{*}$ of these transient highfrequency impedances under multiple high-frequency points is calculated in the (12) to reduce error. Symbol $R_{i}$ is used to mark the comparison result: the measuring unit which holds the minimum high-frequency impedance sets $R_{i}=1$, indicating the No. $i$ DC line may contain a potential fault point.

$$
\left\{\begin{array}{l}
R_{i}=\left\{\begin{array}{l}
1, \forall A_{j}^{*}>A_{i}^{*}, j \neq i \\
0, \exists A_{j}^{*}<A_{i}^{*}, j \neq i
\end{array}\right. \\
A_{i}^{*}=\sqrt{\sum_{k=1}^{K}\left|Z_{i}^{*}\left(\omega_{k}\right) / \omega_{k}\right|^{2} / K}
\end{array}\right.
$$

In accordance with the previous analysis, in the case where there are only two branches connected with the DC bus, the transient high-frequency impedances of these two measuring units will be equal. The fault identification criterion in (12) will not work under this circumstance. However, considering the value of transient high-frequency impedances are different when the fault is located at different branches, the faulted line can be distinguished by comparing the theoretical value of transient high-frequency impedance with the measured one, as shown by formula (13):

$$
\left\{\begin{array}{l}
R_{i}=\left\{\begin{array}{l}
1, A_{i}^{*} \in\left(K_{l} A_{i}, K_{h} A_{i}\right) \\
0, A_{i}^{*} \notin\left(K_{l} A_{i}, K_{h} A_{i}\right)
\end{array}\right. \\
A_{i}=\sqrt{\sum_{k=1}^{K}\left|Z_{i}\left(\omega_{k}\right) / \omega_{k}\right|^{2} / K}
\end{array}\right.
$$

where $Z_{i}\left(\omega_{k}\right)$ denotes the theoretical transient high-frequency impedance at the high frequency point $\omega_{k}$.

Comparing with the (12), the high-frequency impedance $Z_{i}\left(\omega_{k}\right)$ in (13) is obtained from the actual system parameter. The measuring units which conforming the theoretical value (with the reliable coefficient $k_{l}$ and $k_{h}$ ) will set its $R_{i}=1$ to flag the fault direction. For instance, with regard to the measuring No. 9, 10 units in measuring device group (4), the theoretical values of $Z_{i}\left(\omega_{k}\right)$ can be respectively calculated by (10) and (11) when the fault occurs at $f_{3}$ and $f_{4}$. Subscript $A_{i}$ is the RMS of these theoretical transient high-frequency impedances. The $K_{h}\left(K_{h}>1\right)$ and $K_{l}\left(K_{l}<1\right)$ are reliable coefficients.

Fault direction can be identified by measuring the transient high-frequency impedance which is only related to the system structure/parameters and stable. Traditional fault characteristic detection based protection is driven by the converters and DC loads, however the proposed method is driven by the highfrequency source which is located at the fault point. The highfrequency impedance can be different when the more loads are connected. However, the proposed method is based impedance comparison and the results will not change once the system's configuration is fixed. The proposed method is immune to the flexible power flow and the oscillations of the fault transient currents/voltages in DC system.

To carry out faulted line identification, the directional pilot scheme is adopted to protect the full-length of the DC line: when the No. $i$ measuring unit detects that the fault is on its connected line, its $R_{i}$ is set to ' 1 ' and will be sent to the other end of DC line. Only when the measuring units deployed on both ends of the DC line all determine that the fault is located at their connected line, converters will be blocked and trigger the isolators at both ends of the faulted line after fault current is extinguished by the CDSM-MMC. The logical expression mentioned above can be expressed by (13), in which $M, N$ denote the two ends of DC line.

$$
S=\left\{\begin{array}{l}
1, R_{M} \cap R_{N}=1 \\
0, R_{M} \cap R_{N}=0
\end{array}\right.
$$

For the proposed protection, only a logic signal needs to be delivered via the communications, so the data synchronization between the two measuring device groups is unnecessary. The proposed method could protect the full length of the DC lines with the very low-bandwidth requirement.

For the fault behind the DC/DC converters which is located at the terminal of DC distribution network, the fault currents will flow from the converter to the PVs[38] and it can be detected by the directional protection with over-current criteria.

\section{B. Flow Chart of the Proposed Protection}

According to the fault identification criterion, the flow chart of the proposed protection is shown in the Fig. 5, and includes the three steps: (1) Protection Startup; (2) Fault Detection; (3) Faulted Line Selection. With reference to the configuration of the protection in high-voltage direct current (HVDC) system, the principles $d u / d t$ and $d i / d t$ are used as the start-up criterion for the proposed protection. The under-voltage/over-current principle is used to detect the pole to pole fault of the DC line. The proposed transient high-frequency impedance comparison based protection will then be used to carry out the faulted line selection. When both the protection startup and fault detection criterions are satisfied, the converters will be blocked to clear the fault currents. The isolators installed on both ends of the faulted line will then be triggered to isolate the fault point after short-circuit current is extinguished by converter. Following this, the converter can be restarted, and the non-fault area of the DC distribution network will restore the power supply. 


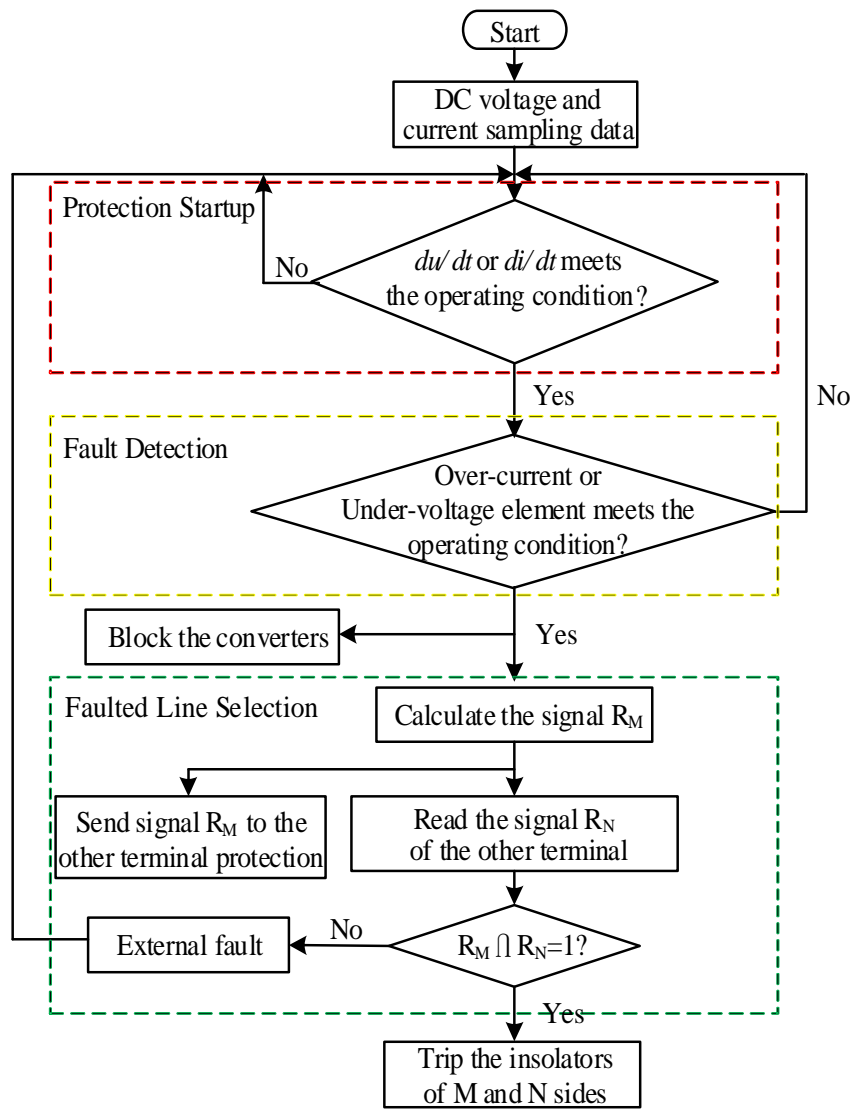

Fig. 5. Flow chart of the proposed protection scheme

\section{SimUlATIONS AND VERIFICATIONS}

In this section, the simulation model of the typical flexible DC system in Fig. 1 is built in PSCAD/EMTDC to verify the effectiveness of the proposed protection. The system includes two independent AC systems $(10 \mathrm{kV})$ connected via a $3.5 \mathrm{~km}$ DC line. Two CDSM-MMCs (5 MVA) operate at the constant DC voltage mode and the constant active/reactive power mode respectively to carry out power conversion and transmission. In addition, the photovoltaic arrays $(2.5 \mathrm{MW})$ and DC loads (5 MW) are integrated with the DC system via DC/DC converter. Simulation step is $10 \mu$ s and the sampling frequency is $50 \mathrm{kHz}$ to provide results with good SNR in the interested frequency range and also is within the data processing limitation of the most signal processing boards, the fault duration time is $0.8 \mathrm{~s}$. A more detailed outline of the simulation model parameters is presented in the Appendix.

Continuous wavelet transformation is applied to extract the high-frequency signals for the protection algorithms proposed in this paper. The Morlet wavelet [39] is selected as mother wavelet in the (15). Where the bandwidth frequency $f_{b}$ is 10 $\mathrm{Hz}$ and the center frequency $f_{c}$ is $2 \mathrm{~Hz}$ to emphasize the accuracy of results in interested high-frequency range. Data measurement window is $5 \mathrm{~ms}$ (respectively $2.5 \mathrm{~ms}$ before and after the fault) to accurately extract the fault transient process, however it is not the rigid requirement and can be modified according the extraction effects.

The algorithms of the proposed protection are computed in MATLAB R2014b and the computer is equipped with Core i5
CPU (3.4GHz) and 4.0 GB RAM. For industry application, the field-programmable gate-array (FPGA) unit is used to process the measuring data and send the protection trigger signal. The total operation time will only take a few milliseconds after the DC fault inception.

$$
\varphi(x)=\frac{1}{\sqrt{\pi f_{b}}} e^{j 2 \pi f_{c} x} e^{-x^{2} / f_{b}}
$$

Considering that the transient impedances of the MMC will be linear to the frequency in high frequency band, the RMS of high-frequency impedances at 1000, 1100, 1200, 1300, 1400, and $1500 \mathrm{~Hz}$ are calculated using (10) and (11) to reduce the measuring error. The selection principle of frequency band is detailed below:

\section{i) The upper limit of the selected frequencies}

The upper limit of the selected frequencies should be lower than half of the data sampling frequency to meet the 'Nyquist sampling theorem. Furthermore, the spectral energy density must be high enough to accurately extract the high-frequency signals when considering the inversely proportional magnitude -frequency characteristic in the Fig. 2(b)(d).

\section{ii) The lower limit of the selected frequencies}

These selected frequencies should satisfy the constraints of the (4). In this case, the DC-side equivalent model of the converter can be linearized as a constant impedance.

\section{iii) Influence of the converter switching frequency}

Rich harmonics will be present around converter switching frequency and that may cause a large error to the protection algorithm. Thus, the selected frequencies should be far away from the converter switching frequency.

\section{A. Verification of MMC High-frequency Model}

When there is a DC fault, the rich frequency domain signals will be generated on the fault point, and transient DC voltages and the currents flowing through $\mathrm{MMC}$ at frequencies ranging from $0.1 \sim 4 \mathrm{kHz}$ can be obtained in the simulation. Therefore, the DC-side high-frequency impedances under various control strategies, controller parameters, and power outputs of MMC can be calculated by (3) and are presented in Fig. 6. Where kp is the proportional coefficient of the PI controller, $\mathrm{P}$ is the active power output of PQ-mode MMC.

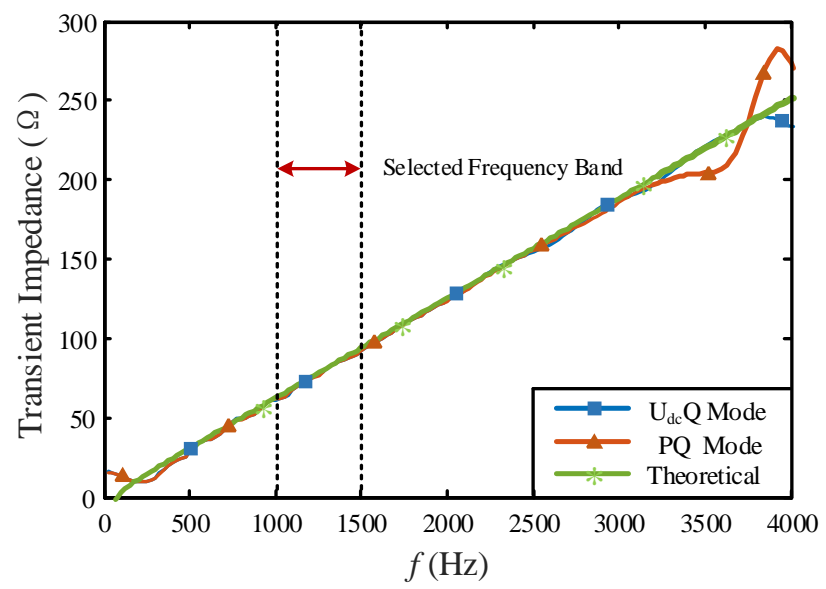

Fig. 6(a). High-frequency impedances under various control strategy 


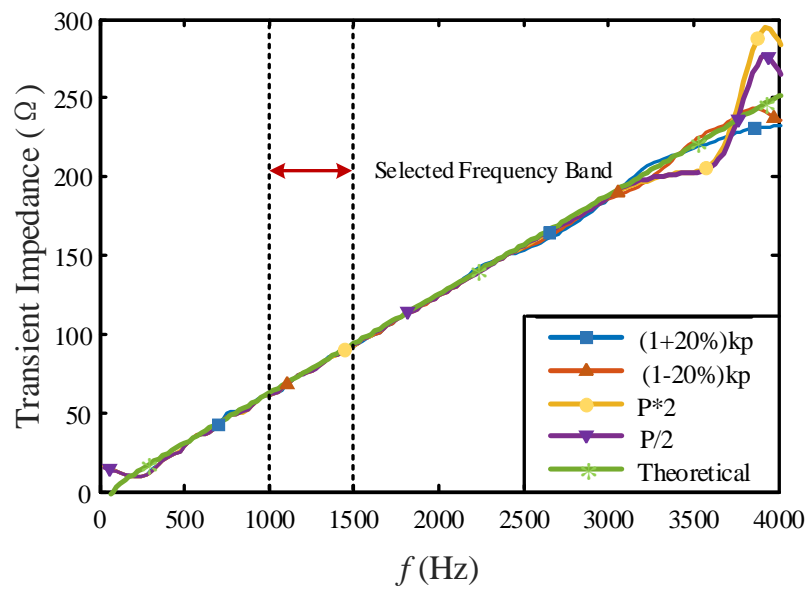

Fig. 6(b). Results under various controller parameters and outputs

As illustrated in the Fig. 6, for the frequencies ranging from $0.5 \sim 3 \mathrm{kHz}$, the measured transient impedances are essentially the same as the theoretical curve calculated by the (5). For frequencies ranging from $3 \sim 4 \mathrm{kHz}$, the difference between the measured value and theoretical value of transient impedance is not obvious (maximum error is about $7 \%$ and average error is less than $3 \%$ ), that is caused by the signal attenuations. In addition, the transient impedances in the high frequency band will display a strong inductive characteristic according to (5) which is independent to the control strategies/parameters of converter. The theoretical analysis corresponds well with the simulation results, verifying the proposed high-frequency impedance model of the MMC.

\section{B. Performance of the Proposed Protection}

To verify the protection algorism, a pole to pole fault is set at $f_{1}$ on DC line1. The transient high-frequency impedance of each measuring unit in measuring device groups (1) and (2) are shown in Fig. 7(a) and (b), respectively. Values $A^{*}$, calculated by (14) of each measuring unit, are provided in Fig. 7(c).

As illustrated in Fig. 7(a)(b), the high-frequency impedance of the No. 2 measuring unit in measuring device group (1) is the minimum at each frequency point in the selected frequency band, and so is the No. 4 measuring unit in device group (2). This finding proves the amplitude of the fault-side transient high-frequency impedance is the lowest in the same measuring device group when multiple lines connect on the DC bus. As $R_{2}$ $=R_{4}=1$, the fault will be located at the DC-line 1 , which is directly connected to measuring unit 2 and 4 , respectively.

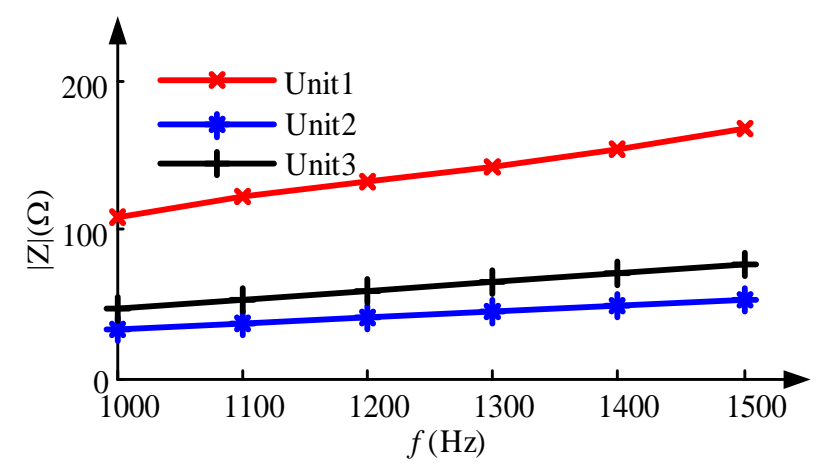

Fig. 7(a). High-frequency impedances of measuring device group (1)

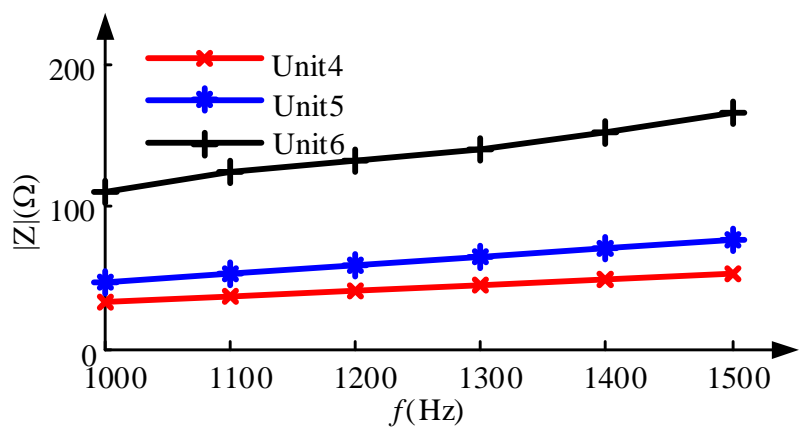

Fig. 7(b). High-frequency impedances of measuring device group (2)

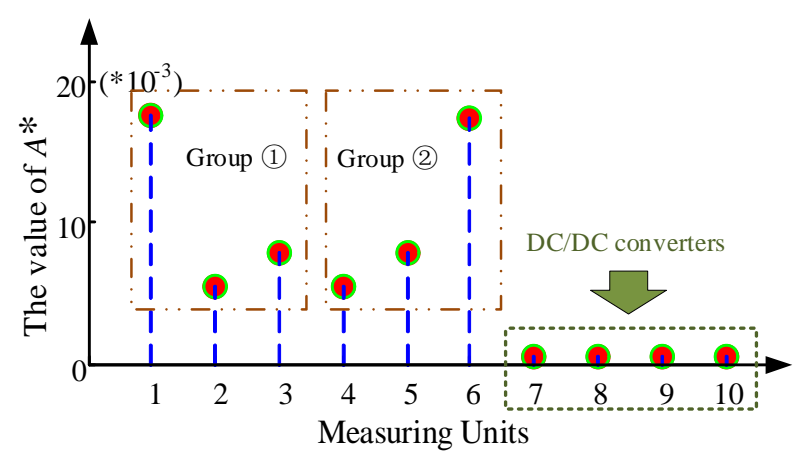

Fig. 7(c). Values $A^{*}$ of each measuring unit when fault is located at $f_{1}$

In addition, for the fault located at DC-line 1, the theoretical transient high-frequency impedance of the No. 7-10 measuring units is the high-frequency impedance of the DC/DC converter. Thus, the values $A^{*}$ of No. 7-10 measuring units will be much smaller than the ones of No. 1-7's, as shown in the Fig. 7(c), which are mainly caused by the low-impedance characteristics of high-voltage smooth capacitors in high-frequency band.

Table I provides the values of $A^{*}$ for the DC fault located at different positions in Fig. 1. The measuring units that conform the fault identification criterion (their $R_{i}$ is set to ' 1 ' to flag the fault direction) have been marked using a dashed box, thus the faulted line can be selected out by applying logical expression in (14). Table I shows that the proposed method completes the faulted line selection reliably via measuring the transient highfrequency impedances in flexible DC systems.

For the measuring device group (1) and (2) deployed on the DC bus with three branches, the fault identification criterion in

TABLE I

Simulation RESUlts When FAUlts ARE LOCATED AT DifFERENT LiNES

\begin{tabular}{|c|c|c|c|c|c|}
\hline Fault location & $f_{1}$ & $f_{2}$ & $f_{3}$ & $f_{4}$ & $f_{5}$ \\
\hline$A_{1}^{*}\left(\times 10^{-3}\right)$ & 17.481 & 2.681 & 9.725 & 9.679 & 9.551 \\
\hline$A_{2}^{*}\left(\times 10^{-3}\right)$ & 5.381 & 7.683 & 7.650 & 7.617 & 2.996 \\
\hline$A_{3}^{*}\left(\times 10^{-3}\right)$ & 7.775 & 4.165 & 4.285 & 4.287 & 4.283 \\
\hline$A_{4}^{*}\left(\times 10^{-3}\right)$ & 5379 & 2.993 & 2977 & 2954 & 7.716 \\
\hline$A_{5}^{*}\left(\times 10^{-3}\right)$ & 7.791 & 4.308 & 4.294 & 4.283 & 4.288 \\
\hline$A_{6}^{*}\left(\times 10^{-3}\right)$ & 17.401 & 9.831 & 9.729 & 9.550 & 9.656 \\
\hline$A_{7}^{*}\left(\times 10^{-3}\right)$ & 0.436 & 0.424 & 0.424 & 0.449 & 0.799 \\
\hline$A_{8}^{*}\left(\times 10^{-3}\right)$ & 0.436 & 0.424 & 0.424 & 0.449 & 0.799 \\
\hline$A_{9}^{*}\left(\times 10^{-3}\right)$ & 0.429 & 0.906 & 0.803 & 8967 & 0.434 \\
\hline$A_{10}{ }^{*}\left(\times 10^{-3}\right)$ & 0.429 & $0.906:$ & 0.803 & 8.967 & 0.434 \\
\hline Results & Line1 & MMC1 & Line3 & $\mathrm{DC} / \mathrm{DC} 1$ & Line2 \\
\hline
\end{tabular}


TABLE II

THEORETICAL HIGH-FREQUENCY IMPEDANCE AND RELIABLE COEFFICIENTS

\begin{tabular}{c|ccc}
\hline Units & $\begin{array}{c}\text { The theoretical } \\
\text { value } A_{i}\left(\times 10^{-3}\right)\end{array}$ & Reliable coefficient & Setting ranges $\left(\times 10^{-3}\right)$ \\
\hline 7 & 0.36 & $K_{l}=0.25 ; K_{h}=4.0$ & $(0.09,1.44)$ \\
8 & 9.3 & $K_{l}=0.80 ; K_{h}=1.2$ & $(7.44,11.16)$ \\
\hline 9 & 9.3 & $K_{l}=0.80 ; K_{h}=1.2$ & $(7.44,11.16)$ \\
10 & 0.36 & $K_{l}=0.25 ; K_{h}=4.0$ & $(0.09,1.44)$ \\
\hline
\end{tabular}

(12) can be used without the threshold settings. However, for the device groups (3) and (4) with only two branches, the threshold criterion should be used. In the practical application, the measuring high-frequency impedances won't strictly equal to their theoretical values due to the measuring errors and the uncertainties of system parameters. The reliability coefficients should be considered when the theoretical value is taken into account during the protection decision. The high-frequency impedance and setting range of each measuring units in group (3) and (4) are provided in Table II.

The measuring errors of the sensors, the computation errors of data processing and the uncertainties of system parameters (such as the impedance errors of DC line) have been taken into account when determining the reliable coefficients. The fault directions could be distinguished by comparing the theoretical value and the measuring value of high-frequency impedances. The measuring units which conform to the setting ranges are marked with a dashed box in the Table.

\section{Influence of Fault Transition Resistance}

For the proposed method, the signal source is located at the fault point. The transient high-frequency impedances are only related to systems own topology/parameter, and are irrelevant to the fault transition resistance. However, the fault resistance will lead to the attenuation of transient high-frequency signal generated by the fault point, and will cause errors in measured high-frequency impedances.

Table III presents the simulation results for DC distribution network in the Fig. 1 , in which the fault occurs at $f_{1}$ and $f_{3}$, respectively, with the transition resistance of $50 \Omega / 100 \Omega$. It can be seen that the proposed method can identify the faulted line reliably, even when the transition resistance reaches up to 100 $\Omega$ in the $\pm 10 \mathrm{kV} \mathrm{DC}$ system. This result shows that the proposed method is robust to fault transition resistance.

TABLE III

SimUlation RESUlts With VARIOUS TRANSITION RESISTANCE

\begin{tabular}{|c|c|c|c|c|}
\hline Fault location & $f_{1}$ & $f_{1}$ & $f_{3}$ & $f_{3}$ \\
\hline $\begin{array}{c}\text { Transition } \\
\text { resistance } / \Omega\end{array}$ & 50 & 100 & 50 & 100 \\
\hline$A_{1}^{*}\left(\times 10^{-3}\right)$ & 17.410 & 24.361 & 22.8143 & 31.237 \\
\hline$A_{2}^{*}\left(\times 10^{-3}\right)$ & 5.402 & 7.424 & 17.940 & 24.795 \\
\hline$A_{3}^{*}\left(\times 10^{-3}\right)$ & 7.773 & 10.700 & 10.030 & 13.661 \\
\hline$A_{4}^{*}\left(\times 10^{-3}\right)$ & 5.372 & 7414 & 7.147 & 10.045 \\
\hline$A_{5}^{*}\left(\times 10^{-3}\right)$ & 7.779 & 10.743 & 10.404 & 14.710 \\
\hline$A_{6}^{*}\left(\times 10^{-3}\right)$ & 17.380 & 23.919 & 22.764 & 31.668 \\
\hline$A_{7}^{*}\left(\times 10^{-3}\right)$ & 0.434 & 0470 & 0.497 & 0001 \\
\hline$A_{8}^{*}\left(\times 10^{-3}\right)$ & 0.434 & 0.470 & 0.497 & 0.801 \\
\hline$A_{9}^{*}\left(\times 10^{-3}\right)$ & 0.429 & 0.491 & 0.399 & 0.433 \\
\hline$A_{10}{ }^{*}\left(\times 10^{-3}\right)$ & 0.429 & :091":4: & :3999: & 0433 \\
\hline Results & Line1 & Line1 & Line3 & Line3 \\
\hline
\end{tabular}

TABLE IV

Simulation Results With NoISE UNDER VARIOUS Signal to NoISE RATIO

\begin{tabular}{|c|c|c|c|c|}
\hline Fault location & $f_{1}$ & $f_{1}$ & $f_{3}$ & $f_{3}$ \\
\hline $\mathrm{SNR} / \mathrm{dB}$ & 30 & 50 & 30 & 50 \\
\hline$A_{1}^{*}\left(\times 10^{-3}\right)$ & 22.035 & 17.622 & 8.4773 & 9.578 \\
\hline$A_{2}^{*}\left(\times 10^{-3}\right)$ & 4.694 & 5.467 & 24.657 & 7.806 \\
\hline$A_{3}^{*}\left(\times 10^{-3}\right)$ & 6.192 & 7.696 & 4.048 & 4399 \\
\hline$A_{4}^{*}\left(\times 10^{-3}\right)$ & 5620 & 5.414 & 1.644 & 3.028 \\
\hline$A_{5}^{*}\left(\times 10^{-3}\right)$ & 10.728 & 7.745 & 3.767 & 4.195 \\
\hline$A_{6}^{*}\left(\times 10^{-3}\right)$ & 19.375 & 16.566 & 9.641 & 10.909 \\
\hline$A_{7}^{*}\left(\times 10^{-3}\right)$ & 1672 & 0385 & 2.150 & 0.564 \\
\hline$A_{8}{ }^{*}\left(\times 10^{-3}\right)$ & 2.859 & 0.544 & 1.835 & 0.366 \\
\hline$A_{9}^{*}\left(\times 10^{-3}\right)$ & 2.616 & 0.467 & 0.798 & 0.809 \\
\hline$A_{10}{ }^{*}\left(\times 10^{-3}\right)$ & 11.965 & 0349 & 1028 & 0792 \\
\hline Results & Line1 & Line1 & Line3 & Line3 \\
\hline
\end{tabular}

\section{Influence of Signal Disturbance}

The transient-impedance characteristics of DC distribution network in high-frequency band is analyzed and is then used to form the proposed fault identification criterion in this paper to improve the high-impedance fault detection. However, the transient high-frequency signal can be disturbed by noise from the sensing instrument itself or in other unpredictable ways. Therefore, it is necessary to evaluate the noise effects on the proposed transient high-frequency impedance measured based protection in this paper.

Simulations are carried out to estimate the influence of the measurement noise by adding Gaussian-noise with different intensity to voltage/current sampling data. The values $A^{*}$ of each measuring unit are provided in Table IV, for when the fault is located at $f_{1}$ and $f_{3}$, respectively. Compared with the simulation results in Table I, it is obvious that the calculation error of $A^{*}$ for each measuring unit increases with added noise. Simulation results show the proposed method can tolerate at least $30 \mathrm{~dB}$ of the signal-to-noise ratio (SNR).

Overall, the noise of the system is not larger than $30 \mathrm{~dB}$, thus the proposed protection is proven to have the satisfactory noise immunity that can reliably achieve faulted line selection under noise circumstances.

\section{CONCLUSIONS}

This paper proposed a transient high-frequency impedance comparison based protection for the flexible DC distribution systems. The main contributions are as follows:

(1) In order to eliminate the effects of unpredictable switching behavior on the time-domain fault characteristics of converter, the control-independent high-frequency impedance model of the MMC-type converters was investigated for the first time in this paper, which can be linearized as a constant impedance in high-frequency band. The converter offers constant impedance regardless of the control strategies, which is the foundation of the network high-frequency impedance analysis in MMC-type DC distribution system.

(2) For the traditional DC protections, the fault characteristics detected by the protection are driven by the converters and DC loads, so the protection is normally sensitive to fault transition resistance. However, for the proposed method, the fault itself is regarded as a signal source located at the fault point, so the 
proposed method is robust to the fault transition resistance.

(3) The deployment of measuring units was only required on the bus side of each branch to form the measuring device group. Complex threshold settings in the case of multiple lines connection were also not required. This is beneficial for the multi-branch system with distributed energy integration.

(4) Although the proposed protection is a pilot communication method, compared with traditional pilot protections (such as the differential protection), the strict data synchronization isn't required, and the high-frequency impedances are only related to the system structure/parameters. Thus, the proposed method is immune to the flexible operating modes of the system.

\section{APPENDIX}

In this paper, DC/DC converters are used to carry out DC voltage matching for photovoltaic arrays and DC loads. The $\mathrm{DC} / \mathrm{DC}$ converter includes the three dual active bridge (DAB) units with the structure of input-parallel/output-series (IPOS) to boost the DC voltage. For each DAB unit, there are two capacitors parallel on the high voltage (HV) and low voltage (LV) sides, respectively, to smooth the DC voltages.

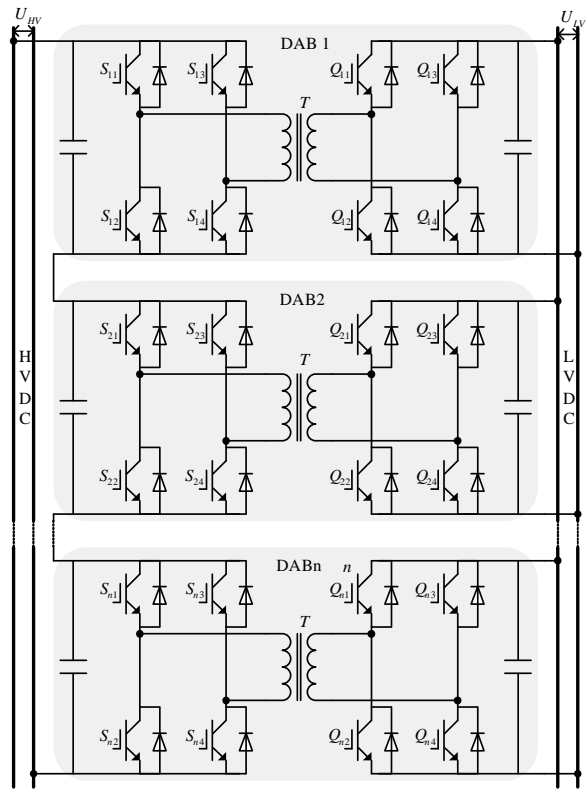

Fig. A1. Detailed structure of the DC/DC converters

The following tables present detailed parameters of the simulation model in Fig. 1.

TABLE A.I

Capacity and Control Scheme of the Converters

\begin{tabular}{cccc}
\hline Converter type & Transformation ratio & Capacity & Control scheme \\
\hline CDSM-MMC1 & AC10kV/DC $\pm 10 \mathrm{kV}$ & $5 \mathrm{MVA}$ & Constant-voltage \\
CDSM-MMC 2 & $\mathrm{AC} 10 \mathrm{kV} / \mathrm{DC} \pm 10 \mathrm{kV}$ & $5 \mathrm{MVA}$ & Constant-power \\
$\mathrm{DC} / \mathrm{DC} 1$ & $\mathrm{DC} \pm 10 \mathrm{kV} / \mathrm{DC} 750 \mathrm{~V}$ & $2.5 \mathrm{MW}$ & $\mathrm{MPPT}$ \\
$\mathrm{DC} / \mathrm{DC} 2$ & $\mathrm{DC} \pm 10 \mathrm{kV} / \mathrm{DC} 750 \mathrm{~V}$ & $5 \mathrm{MW}$ & Constant-power \\
\hline
\end{tabular}

TABLE A.II

Detailed Parameters OF THE DC Lines IN SimUlations

\begin{tabular}{ccccc}
\hline DC line & $\begin{array}{c}\text { Length } \\
(\mathrm{km})\end{array}$ & $\begin{array}{c}\text { Resistance } \\
(\Omega / \mathrm{km})\end{array}$ & $\begin{array}{c}\text { Inductance } \\
(\mathrm{mH} / \mathrm{km})\end{array}$ & $\begin{array}{c}\text { Capacitance } \\
(\mathrm{F} / \mathrm{km})\end{array}$ \\
\hline Line1 & 3.5 & 0.0669 & 0.6857 & $8.34 * 10^{-9}$ \\
Line2 & 1.75 & 0.0669 & 0.6857 & $8.34 * 10^{-9}$ \\
Line3 & 1.75 & 0.0669 & 0.6857 & $8.34 * 10^{-9}$ \\
\hline
\end{tabular}

TABLE A.III

DetaILED PARAMETERS OF THE MMC AND DC/DC CONVERTERS

\begin{tabular}{ccc}
\hline \multirow{3}{*}{ MMC } & The bridge-arm inductance $L_{0}$ & $15 \mathrm{mH}$ \\
\cline { 2 - 3 } & The sub-module capacitance $C_{0}$ & $300 \mu \mathrm{F}$ \\
\cline { 2 - 3 } & The output voltage level number & 11 \\
\hline \multirow{2}{*}{ DC/DC } & The high-voltage side capacitance & $500 \mu \mathrm{F}$ \\
& The low-voltage side capacitance & $150 \mu \mathrm{F}$ \\
\hline
\end{tabular}

\section{REFERENCES}

[1] J. Yu, J. Yu, Y. Wang, et al, "Passivity-based Active Stabilization for DC Microgrid Applications," in CSEE Journal of Power and Energy Systems, vol. 4, no. 1, pp. 29-38, Mar. 2018.

[2] M. E. Baran and N. R. Mahajan, "DC Distribution for Industrial Systems: Opportunities and Challenges," in IEEE Trans. on Industry Applications, vol. 39, no. 6, pp. 1596-1601, Nov-Dec. 2003.

[3] M. Starke, L. M. Tolbert and B. Ozpineci, "AC vs. DC Distribution: A Loss Comparison," 2008 IEEE/PES Transmission and Distribution Conference and Exposition, Chicago, IL, 2008, pp. 1-7.

[4] M. Tabari and A. Yazdani, "Stability of a de Distribution System for Power System Integration of Plug-In Hybrid Electric Vehicles," in IEEE Trans. on Smart Grid, vol. 5, no. 5, pp. 2564-2573, Sept. 2014.

[5] Y. Li, L. He, F. Liu, C. Li, Y. Cao and M. Shahidehpour, "Flexible Voltage Control Strategy Considering Distributed Energy Storages for DC Distribution Network," in IEEE Trans. on Smart Grid, vol. 10, no. 1, pp. 163-172, Jan. 2019.

[6] M. Li, K. Jia, T. Bi and Q. Yang, "Sixth harmonic-based fault location for VSC-DC distribution systems," in IET Generation, Transmission \& Distribution, vol. 11, no. 14, pp. 3485-3490, Sep. 2017.

[7] D. Jovcic, M. Taherbaneh, J. Taisne and S. Nguefeu, "Offshore DC Grids as an Interconnection of Radial Systems: Protection and Control Aspects," in IEEE Trans. on Smart Grid, vol. 6, no. 2, pp. 903-910, Mar. 2015.

[8] Y. Xu, J. Liu and Y. Fu, "Fault-line Selection and Fault-type Recognition in DC Systems Based on Graph Theory," in Protection and Control of Modern Power Systems, vol. 3, no. 3, pp. 267-276, Aug. 2018.

[9] M. Rahman, L. Xu, L. Yao, "Protection of Large Partitioned MTDC Networks Using DC-DC Converters and Circuit Breakers," in Protection and Control of Modern Power Systems, vol. 1, no. 1, pp. 170-178, Dec. 2016.

[10] K. Jia, E. Christopher, D. Thomas, M. Sumner and T. Bi, “Advanced DC Zonal Marine Power System Protection," in IET Generation Transmission \& Distribution, Vol. 8, no. 2, pp. 301-309, Feb. 2014.

[11] M. E. Baran and N. R. Mahajan, "Overcurrent Protection on Voltage Source Converter Based Multi-terminal DC Distribution Systems," in IEEE Trans. on Power Delivery, vol. 22, no. 1, pp. 406-412, Jan. 2007.

[12] D. Salomonsson, L. Soder and A. Sannino, "Protection of Low-Voltage DC Microgrids," in IEEE Trans. on Power Delivery, vol. 24, no. 3, pp. 1045-1053, July 2009.

[13] S. D. A. Fletcher, P. J. Norman, S. J. Galloway and G. M. Burt, "Analysis of the Effectiveness of Non-unit Protection Methods Within DC Microgrids," IET Conference on Renewable Power Generation (RPG 2011), Edinburgh, 2011, pp. 1-6.

[14] A. Meghwani, S. C. Srivastava and S. Chakrabarti, "A Non-unit Protection Scheme for DC Microgrid Based on Local Measurements," in IEEE Trans. on Power Delivery, vol. 32, no. 1, pp. 172-181, Feb. 2017.

[15] M. Farhadi and O. A. Mohammed, "Event-Based Protection Scheme for a Multiterminal Hybrid DC Power System," in IEEE Trans. on Smart Grid, vol. 6 , no. 4, pp. 1658-1669, July 2015.

[16] R. Li, L. Xu and L. Yao, "DC Fault Detection and Location in Meshed Multiterminal HVDC Systems Based on DC Reactor Voltage Change Rate," in IEEE Trans. on Power Delivery, vol. 32, no. 3, pp. 1516-1526, June 2017.

[17] X. Feng, L. Qi and J. Pan, "A Novel Fault Location Method and Algorithm for DC Distribution Protection," in IEEE Trans. on Industry Applications, vol. 53, no. 3, pp. 1834-1840, May-June 2017.

[18] M. K. Bucher and C. M. Franck, "Contribution of Fault Current Sources in Multiterminal HVDC Cable Networks," in IEEE Trans. on Power Delivery, vol. 28, no. 3, pp. 1796-1803, July 2013.

[19] X. Liu, A. H. Osman and O. P. Malik, "Hybrid Traveling Wave/Boundary Protection for Monopolar HVDC Line," in IEEE Trans. on Power Delivery, vol. 24, no. 2, pp. 569-578, April 2009.

[20] K. A. Saleh, A. Hooshyar and E. F. El-Saadany, "Ultra-High-Speed Travelling-Wave-Based Protection Scheme for Medium-Voltage DC Microgrids," in IEEE Trans. on Smart Grid, vol. 10, no. 2, pp. 1440-1451, Oct. 2017.

[21] Y. Zhang, N. Tai and B. Xu, "Fault Analysis and Traveling-Wave Protection Scheme for Bipolar HVDC Lines," in IEEE Trans. on Power Delivery, vol. 27, no. 3, pp. 1583-1591, July 2012 
[22] S. D. A. Fletcher, P. J. Norman, K. Fong, S. J. Galloway and G. M. Burt, "High-Speed Differential Protection for Smart DC Distribution Systems," in IEEE Trans. on Smart Grid, vol. 5, no. 5, pp. 2610-2617, Sept. 2014.

[23] C. Yuan, M. A. Haj-ahmed and M. S. Illindala, "Protection Strategies for Medium-Voltage Direct-Current Microgrid at a Remote Area Mine Site," in IEEE Trans. on Industry Applications, vol. 51, no. 4, pp. 2846-2853, July-Aug. 2015.

24] X. Zheng, N. Tai, J. S. Thorp and X. Yang, "Improved Differential Protection Scheme for Long Distance UHVDC Transmission Line," 2014 IEEE PES General Meeting | Conference \& Exposition, National Harbor, MD, 2014, pp. $1-5$.

[25] M. Monadi, C. Koch-Ciobotaru, A. Luna, J. Ignacio Candela and P. Rodriguez, "Multi-terminal Medium Voltage DC Grids Fault Location and Isolation," in IET Generation, Transmission \& Distribution, vol. 10, no. 14, pp. 3517-3528, Nov. 2016.

[26] G. Song, X. Chu, S. Gao, X. Kang and Z. Jiao, "A New Whole-Line QuickAction Protection Principle for HVDC Transmission Lines Using One-End Current," in IEEE Trans. on Power Delivery, vol. 30, no. 2, pp. 599-607, Apr. 2015.

[27] J. Liu, C. j. Fan and N. 1. Tai, "A Novel Pilot Directional Protection Scheme for HVDC Transmission Line Based on Specific Frequency Current," 2014 International Conference on Power System Technology, Chengdu, 2014, pp. 976-982.

[28] J. Liu, N. Tai, C. Fan and Y. Yang, "Transient Measured Impedance-based Protection Scheme for DC Line Faults in Ultra high-voltage Direct-current System," in IET Generation, Transmission \& Distribution, vol. 10, no. 14, pp. 3597-3609, Nov. 2016.

[29] K. Jia, T. Bi, Z. Ren, D. W. P. Thomas and M. Sumner, "High Frequency Impedance Based Fault Location in Distribution System With DGs," in IEEE Trans. on Smart Grid, vol. 9, no. 2, pp. 807-816, Mar. 2018.

[30] K. Jia, Z. Ren, L. Li, Z. Xuan, and D. Thomas, "High-frequency Transient Comparison Based Fault Location in Distribution Systems With DGs," in IET Generation, Transmission \& Distribution, vol. 11, no. 16, pp. 4068-4077, Nov. 2017.

[31] Y. M. Yeap, N. Geddada, K. Satpathi and A. Ukil, "Time- and FrequencyDomain Fault Detection in a VSC-Interfaced Experimental DC Test System,' in IEEE Trans. on Industrial Informatics, vol. 14, no. 10, pp. 4353-4364, Oct. 2018.

[32] K. Satpathi, Y. M. Yeap, A. Ukil and N. Geddada, "Short-Time Fourier Transform Based Transient Analysis of VSC Interfaced Point-to-Point DC System," in IEEE Trans. on Industrial Electronics, vol. 65, no. 5, pp. 40804091, May 2018

[33] K. D. Kerf et al., "Wavelet-based Protection Strategy for DC Faults in Multiterminal VSC HVDC Systems," in IET Generation, Transmission \& Distribution, vol. 5, no. 4, pp. 496-503, Apr. 2011.

[34] D. Tzelepis et al., "Centralised Busbar Differential and Wavelet-based Line Protection System for Multi-terminal Direct Current Grids With Practical IEC61869-compliant measurements," in IET Generation, Transmission \& Distribution, vol. 12, no. 14, pp. 3578-3586, Oct. 2018.

[35] Z. Wang and R. S. Balog, "Arc Fault and Flash Signal Analysis in DC Distribution Systems Using Wavelet Transformation," in IEEE trans. on Smart Grid, vol. 6, no. 4, pp. 1955-1963, July 2015

[36] Y. Xue and Z. Xu, "On the Bipolar MMC-HVDC Topology Suitable for Bulk Power Overhead Line Transmission: Configuration, Control, and the DC Fault Analysis," in IEEE Trans. on Power Delivery, vol. 29, no. 6, pp. 2420-2429, Dec. 2014.

[37] G. Tang, Z. Xu, Y. Zhou, "Impacts of Three MMC-HVDC Configurations on AC System Stability Under DC Line Faults," in IEEE Trans. on Power System, vol. 29, no. 6, pp. 3030-3040, Nov. 2014.

[38] K. Jia, H. Wei, T. Bi, D. Thomas and M. Sumner, "An Islanding Detection Method for Multi-DG Systems Based on High Frequency Impedance Estimation," in IEEE Trans. on Sustainable energy, Vol. 8, no. 1, pp. 74-83, Jan. 2017.

[39] J. A. Proakis, "Digital Signal Processing Principles, Algorithms, and Applications, 4th ed,” Upper Saddle River, NJ, USA: Prentice-Hall, 2007.

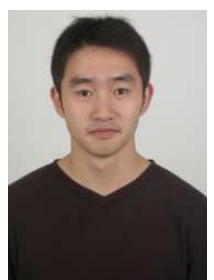

Ke Jia was born in China in 1986. He received MSc and the Ph.D. degree in electrical engineering from Nottingham University, U.K., in 2008, and 2011, respectively and then worked as a Research Fellow until 2013. He is currently an Associate Professor at the North China Electric Power University. His research interests include power system protection and fault location, microgrid automation and renewable energy.

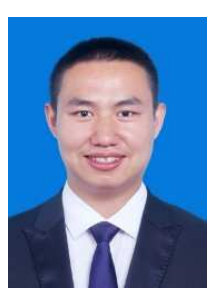

Zhenwen Xuan was born in China in 1993. He received Master's degree in electrical engineering from North China Electric Power University, Beijing, in 2019. $\mathrm{He}$ is currently an electrical engineer at the State Grid Beijing Electric Power Research Institute. His research interests include power system control and protection, micro-grid and renewable energy.

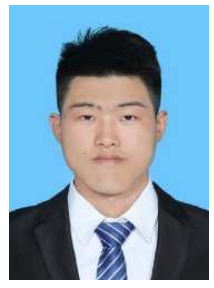

Tao Feng was born in China in 1993. He received bachelor's degree in electrical engineering from South China Agricultural University, Guangzhou, in 2016. He is currently pursuing the master's degree in electrical engineering with North China Electric Power University. His research interests include power system protection and control.

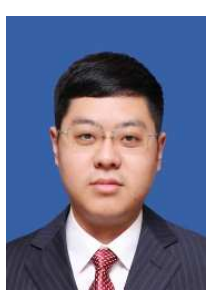

Congbo Wang was born in China in 1990. He received MSc in electrical engineering from Northeast Electric Power University, Jilin, in 2016. He is currently a doctor candidate in Electrical Engineering at North China Electric Power University. His research interests include power system protection and control, fault location and renewable energy.

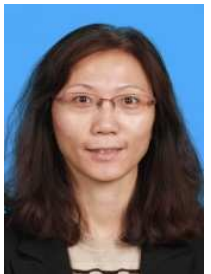

Tianshu Bi (M'1998, SM'09) received her Ph.D. degree at the Department of EEE in the University of Hong Kong in 2002. She is currently a professor at North China Electric Power University. Her research interests include power system protection and control, synchronized phasor measurement technique and its application.

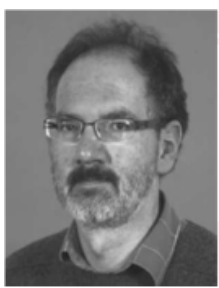

David W. P. Thomas (M'94-SM'09) received the B.Sc. degree in physics from Imperial College of Science and Technology, London, U.K., the M.Phil. degree in space physics from Sheffield University, Sheffield, U.K., and the Ph.D. degree in electrical engineering from Nottingham University, Nottingham, U.K., in 1981, 1987, and 1990, respectively. In 1990, he joined the Department of Electrical and Electronic Engineering, University of Nottingham, Nottingham, as a Lecturer, where he is currently an Associate Professor and Reader. His research interests include power system transients, power system protection, electromagnetic compatibility, and electromagnetic simulation. Dr. Thomas is a member of CIGRE and a Convenor for CIGRE JWG 4.207. He is also vice-chair for IEEE EMC Technical committee 7 "Low frequency EMC". 